\title{
Diffusion model of photoaligning in azo-dye layers
}

\author{
Vladimir Chigrinov, Sergey Pikin,* Andrey Verevochnikov, Vladimir Kozenkov, Maxim Khazimullin, Jacob Ho, \\ Dan Ding Huang, and Hoi-Sing Kwok \\ Hong Kong University of Science and Technology, Clear Water Bay, Kowloon, Hong Kong
}

(Received 13 January 2004; published 18 June 2004)

\begin{abstract}
The model of the rotational diffusion of the azo-dye molecules under the action of polarized uv light was used to explain the formation of the photoinduced order in azo-dye layers. We consider both the approximations of negligible and strong molecular interaction during the process of the reorientation under the field of a polarized light. We constructed an experimental setup, based on a photoelastic modulator, that allows accurate in situ measurements of the phase retardation $\delta$ of thin film as a function of the exposure time $t_{\exp }$ and exposure power $W\left(\mathrm{~W} / \mathrm{cm}^{2}\right)$. A good agreement with experiment was observed. Fitting the experimental curves $\delta\left(t_{\exp }\right)$ for different power values $W$, we can estimate the coefficient of rotational diffusion $D$, azo-dye order parameter $S\left(t_{\text {exp }}\right)$, and other parameters of the rotational diffusion model.
\end{abstract}

DOI: 10.1103/PhysRevE.69.061713

PACS number(s): 61.30.Hn, 61.30.Gd

\section{INTRODUCTION}

Recently, we have shown the possibility of obtaining high-quality, noncontact photoalignment layers using the azo-dye structures [1-3]. Photochemical stable azo-dye layers are an example of pure reorientation of the absorption oscillator of the azo-dye molecules perpendicular to the polarization of the activating uv light. The azo-dye molecules that have their transition dipole moments parallel to the direction of the polarized light will most probably get the excess energy, which results in their reorientation from the initial position. This process can be described statistically as a diffusion motion of the dye molecules under the action of the polarized light $[4,5]$. This results in an excess of chromophores with the absorption oscillator perpendicular to the polarization of the uv light. Hence, a thermodynamical equilibrium in the new oriented state will be established. Consequently, the anisotropic dichroism and optical retardation are photoinduced permanently and the associated order parameter as a measure of this effect can be very large in some of these materials. The photochemical mechanisms, such as cross linking, photodegradation, and even cis-trans isomerization are most likely avoided in this case $[1,6]$.

In this paper we will discuss the model of rotational diffusion of the azo-dye molecules under the action of polarized uv light and show that experimental time behavior of photoinduced birefringence in the azo-dye thin films can be well described by this model. Previous theoretical investigations of azo-dyes photoinduced birefringence were mostly based on taking into account the photochemical transformations such as cross linking in poly (vinyl)cinnamate derivatives and photodegradation in polyimides [7-14] or cis-trans photoisomerization process in azobenzene side-chain polymers [15-17]. A similar model has already been applied to describe the photoinduced birefringence in azo-dye Langmuir Blodgett films [18].

*On leave from Shubnikov's Institute of Crystallography, Russian Academy of Sciences, Moscow, 119333, Russia.
The pure azo-dye layers can also be used as photoaligning agents in liquid crystal display (LCD) cells $[1-3,19,20]$. The thermal and uv stability of the photoaligned azo-dye layers is high and can be considerably improved after thermopolymerization of azo-dyes layers [20]. The azo-dye layers exhibit a sufficiently high anchoring energy of liquid crystal with the substrate, which makes them one of the most attractive photoaligning materials for LCD technology [21].

\section{DIFFUSION MODEL}

\section{A. General consideration}

Azobenzene sulfuric dye SD, used in the experiment, is shown in Fig. 1. When the azo-dye molecules are optically pumped by a polarized light beam, the energy absorbed for the transformation is proportional to the square of the cosine $\theta$, the angle $\theta$ characterizing the orientation of dye molecule
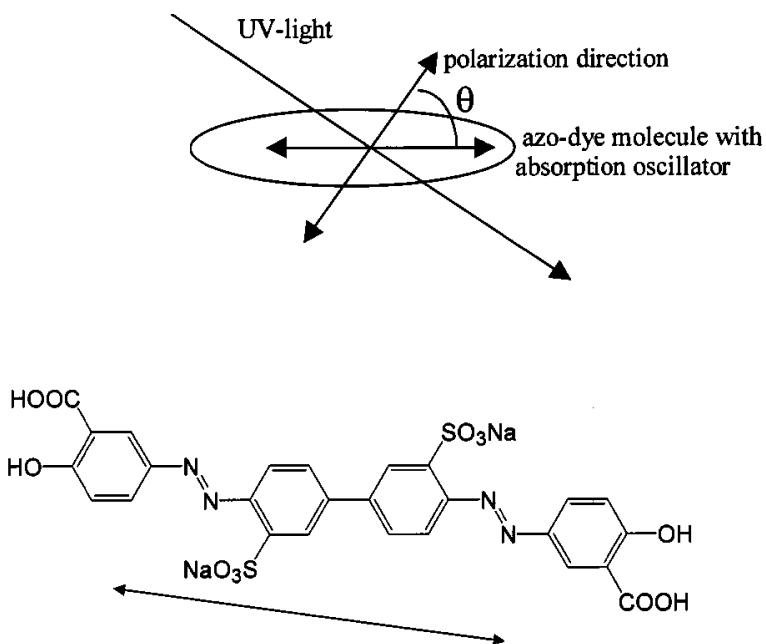

Azo-dye absorption oscillator (chromophore)

FIG. 1. The sulfuric azo-dye SD. Under the action of polarized light the SD molecules tend to reorient perpendicular to the polarization of the activated light. The absorption oscillator is parallel to the molecular axis. 
with respect to the polarization vector of light (Fig. 1). In other words, this probability distribution is nonuniform and has an angular dependence. Therefore, the azo-dye molecules that have their transition dipole moments parallel to the direction of the polarized light will most probably get the excess energy, which results in their reorientation from the initial position. This gives an excess of chromophores in a direction at which the absorption oscillator is perpendicular to the polarization of the uv light (Fig. 1).

If rotating molecules are at every instant of time in a thermal equilibrium, and the rotation takes place in the field with a potential energy $U=\Phi k T$, where $T$ is an absolute temperature, $k$ is the Boltzmann constant, and $\Phi$ is relative potential energy, the equation of Brownian orientational diffusion, also known as the Boltzmann-Smoluchowski equation, can be used to describe the process of the orientational diffusion, e.g., optical Kerr effect in initially isotropic liquids $[4,22,23]$ or rotatory diffusion of solutions of rod-like macromolecules [24]. For rod-like azo-dye molecules with a cylindrical symmetry the only coordinate will be a polar angle $\theta$, the angle between the molecular absorption oscillator and the direction of the polarization of the activating light (Fig. 1). The potential energy $U=\Phi k T$ of the activating light will also be the function of the angle $\theta$ only, i.e., $\Phi=\Phi(\theta)$ and the equation of orientational diffusion is $[4,22,23]$

$$
\begin{aligned}
& \frac{\partial^{2} f}{\partial \theta^{2}}+\frac{\partial}{\partial \theta}\left(f \frac{\partial \Phi}{\partial \theta}\right)=\frac{1}{D} \frac{\partial f}{\partial t}, \\
& \Phi=\frac{A}{2} \cos ^{2} \theta, A=\frac{I \alpha V_{M} \tau}{k T} .
\end{aligned}
$$

Here, we assume that the potential energy $\Phi k T$ is proportional to the light intensity at the direction of the absorption oscillator, i.e., the number of photons absorbed by azo-dye molecule. The energy of photons is not wasted on the change of the molecular conformation or luminescence, and is responsible for the rotation of the azo-dye molecule from its initial random position to the direction perpendicular to the activating light polarization [1].

The order parameter is determined as the thermodynamic average $\left\langle P_{2}(\theta)\right\rangle$

$$
\begin{gathered}
P_{2}(\theta)=\frac{1}{2}\left(3 \cos ^{2} \theta-1\right), \\
\left\langle P_{2}\right\rangle=\int_{0}^{\pi} P_{2}(\theta) f(\theta, t) \sin \theta d \theta / \int_{0}^{\pi} f(\theta, t) \sin \theta d \theta .
\end{gathered}
$$

Here, the probability density function $f(\theta, t)$ describes the movement of molecules in two dimensions, i.e., in a certain plane, where the angle $\theta$ characterizes the orientation of dye molecule with respect to the polarization vector of the activating light. The orientation of the azo-dye molecule in the perpendicular plane is not controlled by light. The value $f(\theta, t) d \theta$ is the number of molecules whose axes are located within the polar angles $(\theta, \theta+d \theta)$ at the unit sphere [25]. Equations (1) and (2) are correct if there is no interaction between azo-dye molecules. Such a situation can take place in very dilute systems with azo-dyes, e.g., in special polymer films having a small amount of azo-dye molecules. Dependent on the sign of the parameter $A$, the molecular orientation occurs either at the angle $\theta=\pi / 2(A>0)$ or $\theta=0, \pi(A<0)$. Equation (3) includes also the light power $I\left(\mathrm{~W} / \mathrm{cm}^{2}\right)$, the absorption coefficient $\alpha(1 / \mathrm{cm})$, and the molecular volume $V_{M}$, absolute temperature $T$, and Boltzmann constant $k$. The parameter $\tau$ is the relaxation time-the time of "cooling" of azo-dye molecule and its surroundings. During this time $\tau$ the azo-dye molecule absorbs the energy of light, overcomes the potential barrier between two orientational states when it interacts with its surroundings, and then "cools down" to the new state. In polymers with a small concentration of azo-dye molecules, the absorption coefficient $\alpha$ is much smaller than in the case of pure azo-dye film, and therefore the parameter $A$ is also small there. The parameter $U=\Phi k T$ $=1 / 2 I \alpha V_{M} \tau \cos ^{2} \theta$ plays the role of a certain potential which tends to make the azo-dye molecule rotate due to the light action, and can be considered as the change of chemical potential per one molecule related to the corresponding change of the free energy. The process of such a reorientation can be considered as isothermal. During this process the free energy of the azo-dye molecules changes due to the light action, and the thermal energy is scattering. We should point out that the change of the free energy takes place for the reoriented molecules only. After this reorientation the molecules are not affected by light again, i.e., this free-energy change per one molecule is a constant value. Only the quantity of reoriented molecules increases with time, and this is described by the variation of the angular distribution function $f$.

\section{B. Estimation of the "cooling time" $\tau$ in the diffusion model}

Unfortunately, the parameter $\tau$ cannot be exactly calculated due to the complicated nature of the numbered interactions and molecular kinetics, but $\tau$ should be much smaller than the time of light exposure to enable the azo-dye molecules to get to a new orientational state. Parameter $\tau$ can be estimated from the experiments on photoaligning. If $k T$ $\sim 10^{-20} \mathrm{~J} \quad$ (room temperature), $\mathrm{A} \sim 10^{5} 1 / \mathrm{cm}, \quad I$ $\sim 10^{-2} \mathrm{~W} / \mathrm{cm}^{2}, V_{M} \sim 10^{-20} \mathrm{~cm}^{3}$, then $\tau \sim 10^{-4}-10^{-3} \mathrm{~s}$ for $A$ $\sim 0.1-1$. The latter limits of the parameters values have been found from the experimental data [1]. We assume, that the characteristic molecular size is about 20-30 $\AA$ (Fig. 1) and estimate the absorption coefficient from the measured optical density $D(D \sim 0.2$ for azo-dye layer thickness of $10 \mathrm{~nm})$ [1]. The values of the coefficient $A \sim 0.1-1$ provide the best fitting of the experimental curves by the diffusion model (see the results below). The time $\tau$ may be dependent on the thermo-conductivity of substance $\kappa$, heat capacity $c_{p}$, density $\rho$, and characteristic size of sample $L$ on which the distribution of temperature becomes homogeneous: $\tau \approx L^{2} c_{p} \rho / \kappa$. Since $\rho \approx 1 \mathrm{~g} / \mathrm{cm}^{3}, \quad c_{p} \approx 1.6 \mathrm{~J} \mathrm{~g}^{-1} \mathrm{~K}^{-1}, \quad \kappa \approx 0.1 \mathrm{~W} \mathrm{~m}^{-1} \mathrm{~K}^{-1}$ [1], we obtain the estimate $\tau \sim 10^{-4} \mathrm{~s}$ for $L \approx 2 \mu \mathrm{m}$.

\section{Diffusion model in case of negligible intermolecular interaction}

Assuming the azimuthal symmetry of the molecular distribution, we can write 


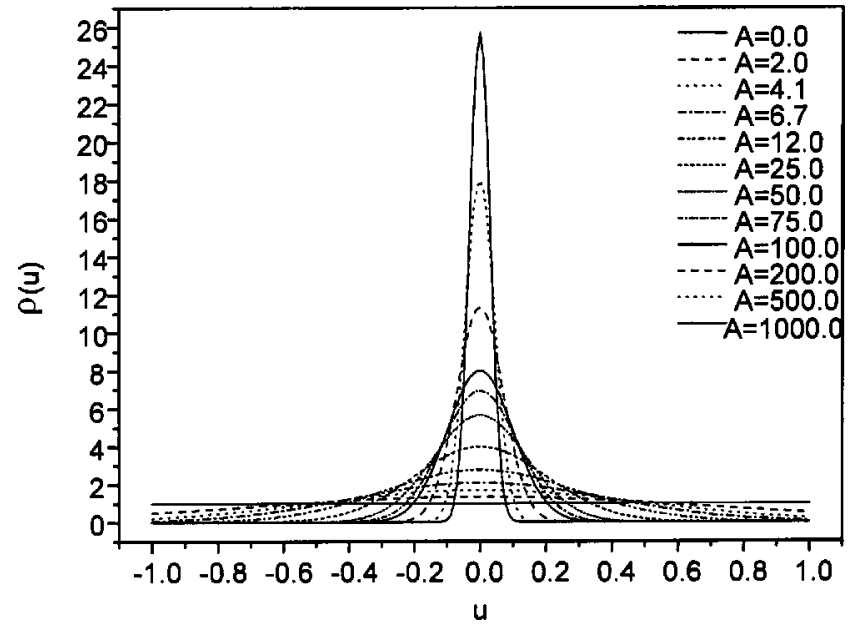

FIG. 2. The distribution function $\rho(u), u=\cos \theta$ of the azo-dye molecules for various values of the parameter $A$, proportional to the intensity of the activated light (2).

$$
f=f(\theta, t)=\rho(u, t) / 2, u=\cos \theta .
$$

Equation (1) can be rewritten as

$$
\begin{aligned}
& \left(1-u^{2}\right) \cdot \partial^{2} \rho / \partial u^{2}-2 u \partial \rho / \partial u+A \rho\left(3 u^{2}-1\right)-A u\left(1-u^{2}\right) \partial \rho / \partial u \\
& \quad=1 / D \partial \rho / \partial u,
\end{aligned}
$$

where

$$
\begin{gathered}
\rho(u, 0)=1, \rho(-u, t)=\rho(u, t), \\
\int_{0}^{\pi} f(\theta) \sin \theta d \theta=\int_{-1}^{1} \rho(u) d u=1,
\end{gathered}
$$

states the initial, symmetry, and normalization condition, respectively.

The saturated $(\mathrm{t} \rightarrow \propto)$ solution of the problem (4) and (5) for $A \gg 1$ and $t \gg 1 / D$

$$
\rho(u)=\sqrt{2 A / \pi} \exp \left(-A u^{2} / 2\right), \mathrm{u} \approx 1 / \sqrt{\mathrm{A}} .
$$

We have also solved the problem (4) and (5) numerically, and showed that the function $\rho(u)$ indeed comes very close to the $\delta$ function for $A \gg 1$ and $t \gg 1 / D$ (Fig. 2)

$$
\rho(u) \rightarrow \delta(u)=\delta(\theta-\pi / 2) .
$$

The perfect order $\theta \equiv \pi / 2, u \equiv 0$ in our case takes place when the absolute value of the order parameter is maximal, $S_{m}=-1 / 2$. Then, the saturated $(t \rightarrow \propto)$ value of the relative order parameter $s=S / S_{m}(0 \leqslant s \leqslant 1)$ can be obtained from (3) and (7)

$$
s \approx 1-3[1-\exp (-A / 2)] / A \int_{0}^{1} \exp \left(-A u^{2} / 2\right) d u
$$

for sufficiently large values of $A \gg 1$.
If $A \ll 1, \rho(u, t)$ can be expanded in a series of Legendre polynomials $P_{n}(u)[4]$ as

$$
\rho(u, t)=1+\sum_{n=1}^{\infty} a_{n}(t) P_{n}(u) \approx 1-A / 3[1-\exp (-6 D t)] P_{2}(u)
$$

The relative order parameter is obtained from (3) and (10) as

$$
s \approx \frac{2 A}{15}\left[1-\exp \left(-\frac{t}{\tau_{\text {rise }}}\right)\right]
$$

or the saturation value of the order parameter is directly proportional to the intensity $I$ of the activating light (2). The response (rise) time

$$
\tau_{\text {rise }}=\frac{1}{6 D} \approx \frac{\pi \eta a_{m}}{k T}
$$

where $D=k T / 6 \pi \eta a_{m}$ is a diffusion coefficient, $\eta$ is the viscosity, $a_{m}$ is the characteristic size of the molecule or molecular cluster. The size of the cluster $a_{m}$ can be dependent on the density (or concentration) of the free and photoaligned azo-dye molecules and can be considered as the effective radius of gyration (the effective hydrodynamic radius) [26]. In this case $a_{m}$ is a function of the exposure energy; however, in the approximation of noninteracting azo-dye molecules, we shall not take this dependence into account. The relation (12) states that the rise time of the effect is independent of the intensity of the activating light.

Looking for the kinetics of the the process of the induced birefringence, we get

$$
\left.\frac{d s}{d t}\right|_{t=0} \approx \frac{2 A}{15 \tau_{\text {rise }}}=\frac{4 A D}{5},
$$

i.e., the rate of the induced order parameter $d s /\left.d t\right|_{t=0}$ is directly proportional to the relative intensity of the activated light.

\section{Introduction of new potential to allow for intermolecular interactions}

In the general case, particularly for dye films, we should take into account the interaction of dye molecules among themselves in the process of the rotational diffusion. This interaction cannot be neglected when the molecular concentration is high [27]. We may use the approximation of mean field acting as the thermodynamic average $\left\langle P_{2}(\theta)\right\rangle$. Thus, the effective potential, in which the dye molecule stays, can be written in the form

$$
U=\frac{1}{2} I \alpha V_{M} \tau \cos ^{2} \theta+a\left\langle P_{2}\right\rangle P_{2}(\theta),
$$

where $\alpha$ is a phenomenological constant. The potential (14a) is the "mean-field" approximation, similar to that proposed in the Maier-Saupe model [28]. The amplitude of the mean field is proportional to the order parameter $S=\left\langle P_{2}\right\rangle$, while the function $P_{2}(\theta)$ makes the interaction potential orientation dependent [29]. 


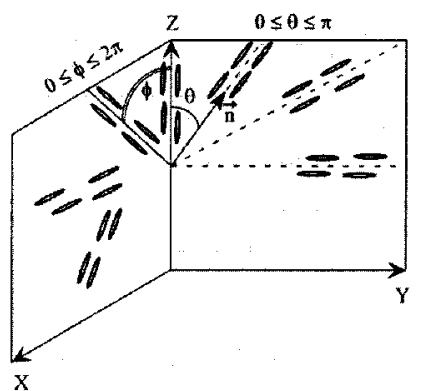

a)

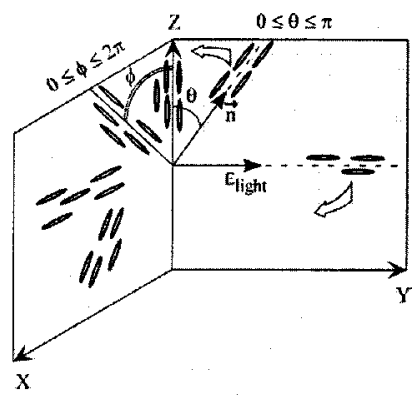

b)

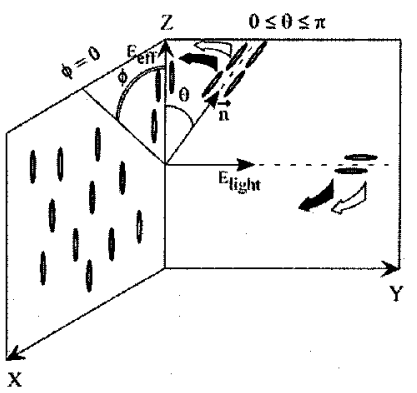

c)

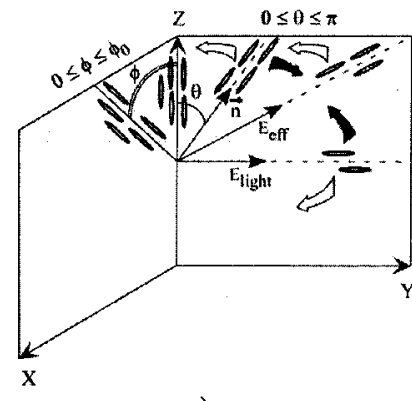

e)

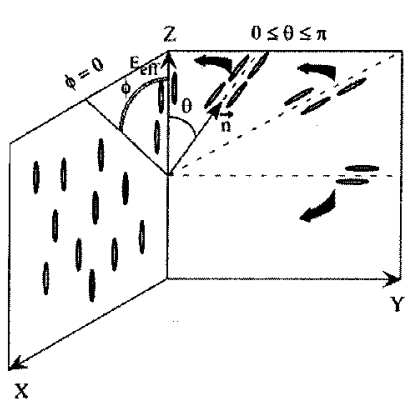

d)

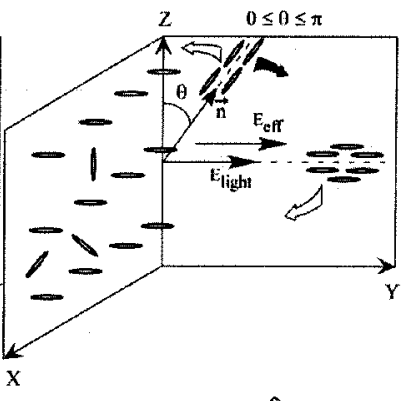

f)

FIG. 3. Distributions of azo-dye molecules, oriented in two planes, as the functions of relative light intensity $A$, temperature $T$, and effective field $E_{\text {eff }}$. The empty and filled arrows correspond to the torques created by polarized light and effective field. $S$-order parameter. (a) $A=0, T>T_{c}, E_{\text {eff }}=0 ; S=0$, isotropic orientation in $x z$. plane; (b) $A \neq 0, T<T_{c}, E_{\text {eff }}=0 ; S \neq 0$, isotropic orientation in $x z$ plane. (c) $A \neq 0, T<T_{c}, E_{\text {eff }} \neq 0 ; S \neq 0$, anisotropic orientation in $x z$ plane. (d) $A=0, T<T_{c}, E_{\text {eff }} \neq 0 ; S \neq 0$, anisotropic orientation in $x z$. plane. (e) $A \neq 0, T<T_{c}, E_{\text {eff }}$ is weak; $S \neq 0$, isotropic orientation in $x z$ plane. (f) $A \neq 0, T>T_{c}$, Eeff is strong; $S \neq 0$, azo-dye molecules are oriented along axis $y$.

If initially, because of the molecular interaction and action of a certain effective electric field $E_{\text {eff }}$, the dye molecules are oriented at a fixed angle $\theta_{0}$ with respect to the polarization of light, then Eq. (14a) should be modified

$$
U=\frac{1}{2} I \alpha V_{M} \tau \cos ^{2} \theta+a\left\langle P_{2}\right\rangle P_{2}\left(\theta-\theta_{0}\right)-\chi E_{\text {eff }}^{2} \cos ^{2}\left(\theta-\theta_{0}\right),
$$

where $\chi$ is the positive dielectric anisotropy. Accordingly, when $I=0$ before the moment $t=0$, the initial function $f_{0}(\theta)=f(\theta, 0)$ is not isotropic in this case, and must be found from the equation of diffusion with potential (14b), where $I=0$. Figures 3(e) and 3(f) show different mutual orientations of the director $\mathbf{n}$ (in the planes $x z$ and $y z$ ), polarization of light $\mathbf{E}_{\text {light }}$ oriented along axis $y$, and effective molecular field $\mathbf{E}_{\text {eff }}$ (in the plane $y z$ ). The initial isotropic distribution of $\mathbf{n}$ for zero values of the effective field and light intensity is shown in Fig. 3(a). The light action induces the rotation of dye molecules mainly in the plane $x z$ [Figs. 3(b) and 3(c)]. In the plane $x z$ the director distribution is either isotropic if $\mathbf{E}_{\text {eff }}=0$ [Fig. 3(b)], or anisotropic if an effective field is present [Fig. 3(c)]. The existence of $\mathbf{E}_{\text {eff }}$ can be related to the existence of an anisotropic substrate, boundaries of individual areas in the film, or some other defects creating an orienting force.

We shall assume, first, that the potential $U$ in the equation of diffusion is given by Eq. (14a), and the initial function $f_{0}(\theta)=1 / 2$, i.e., isotropic. Thus, taking into account (3) and (14a), the equation for the function $f(\theta, t)$ can be written in the form

$$
\frac{\partial^{2} f}{\partial \theta^{2}}-\left(\frac{1}{2} A+\frac{3}{2} \frac{a}{k T}\left\langle P_{2}\right\rangle\right) \frac{\partial}{\partial \theta}[f \sin (2 \theta)]=\frac{1}{D} \frac{\partial f}{\partial t} .
$$

The solution of Eq. (15) can only be found numerically in the whole time interval from $t=0$, when $f(\theta, 0)=1 / 2$ and $S$ $=0$, up to $t \rightarrow \infty$, when certain limits for $f$ and $S$ have to be observed. We can analyze analytically several limit cases which qualitatively show various possibilities of the time behavior of function $f(\theta, t)$ and the order parameter $\left.\left\langle P_{2}(\theta)\right\rangle\right|_{t}$.

\section{E. Kinetics of order parameter $S$ in diffusion model}

$$
\text { 1. The case: } t \rightarrow \infty, \mathbf{A}=0,\left.\left\langle P_{2}\right\rangle\right|_{t \rightarrow \infty}=0
$$

We find the solution $f(\theta, t) \approx f_{0}+\delta(\theta, t)$, where $\delta \ll f_{0}$. Using (5) we obtain the following results:

$$
\begin{gathered}
\frac{1}{D} \frac{\partial \delta}{\partial t}=\frac{\partial^{2} \delta}{\partial \theta^{2}}-\frac{3 a f_{0}}{k T} \cos 2 \theta \int_{0}^{\pi} P_{2} \delta \sin \theta d \theta, \\
\delta(\theta, t)=\exp (-\lambda t) y(\theta), \\
y(\theta)=C \cos 2 \theta, \\
\lambda=D\left(4+\frac{8 a f_{0}}{5 k T}\right), \\
\left.\left\langle P_{2}\right\rangle\right|_{t \rightarrow \infty} \propto \frac{8 C}{15} \exp \left[-D\left(4-\frac{8 f_{0}|a|}{5 k T}\right) t\right],
\end{gathered}
$$

where $f_{0}=\frac{1}{2}, a<0$.

Thus, we see from (16) that the phase transition occurs below the temperature $T_{c}=2 f_{0}|a| / 5 k$, so that $\left.\left\langle P_{2}\right\rangle\right|_{t \rightarrow \infty} \equiv S$ $\neq 0$ at $T<T_{c}$, since the order parameter increases with time at these temperatures in accordance with Eq. (16e). At $T>T_{c}$, the magnitude $\left.\left\langle P_{2}\right\rangle\right|_{t \rightarrow \infty}$ approaches zero by the exponential law with characteristic time $T_{c} /\left[4 D\left(T-T_{c}\right)\right]$, which becomes large (slowing down of relaxation) when the temperature approaches the phase transition point. This situation for $t \rightarrow \infty$ is illustrated by Fig. 3(a). Numerical calculations of the relative order parameter $s$ for $T>T_{c}$, when the light was 


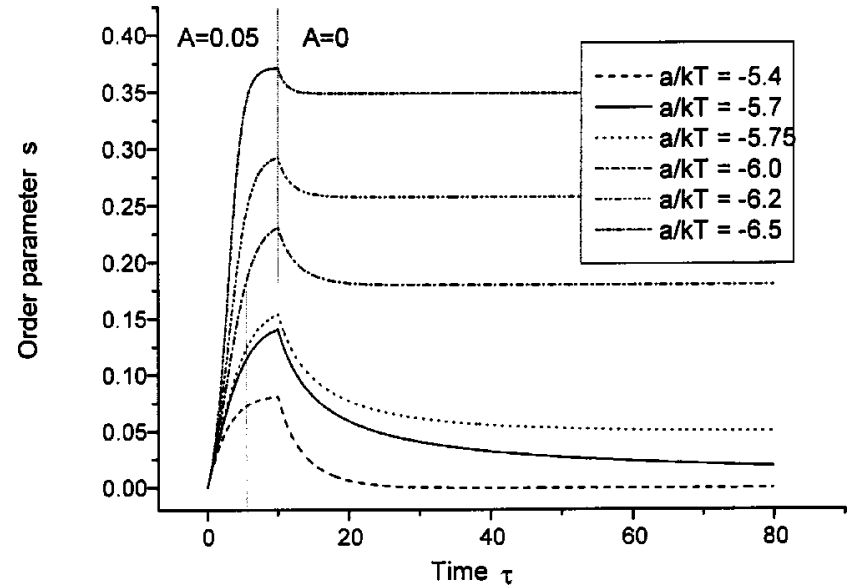

FIG. 4. Relaxation of the relative order parameter $s$ for various values of parameters $A$ and $a / k T$. The time scale is given in relative units $\tau=t D$. The results are obtained by numerical solution of (15).

switched off, are shown in Fig. 4 by two curves: for $a / k T$ $=-5.4, s(t)$ is rapidly decreasing with time; for $a / k T=-5.7$, the described slowing down of the $s(t)$ curve is observed.

\section{The case: $T<T_{c}, A=0$}

We shall find $\left.\left\langle P_{2}\right\rangle\right|_{t \rightarrow \infty} \equiv S \neq 0$. Now, we have

$$
f=f_{0}(\Theta)+\delta(\theta, t), S=\int_{0}^{\pi} P_{2} f_{0} \sin \theta d \theta .
$$

After the substitution of (17) to (15), the equation for function $f_{0}(\theta)$ is

$$
\frac{\partial^{2} f_{0}}{\partial \theta^{2}}+\frac{\partial}{\partial \theta}\left[f_{0}\left(-\frac{3 a}{2 k T} \sin 2 \theta \int_{0}^{\pi} P_{2} f_{0} \sin \theta d \theta\right)\right]=0
$$

which has the solution

$$
f_{0}=\exp \left(-\frac{a}{k T} S P_{2}\right)\left[C_{1}+C_{2} \int_{0}^{\theta} d x \exp \left(\frac{a S}{k T} P_{2}(x)\right)\right] .
$$

Taking into account Eqs. (3) and (19), we obtain the equation for the order parameter $S$

$$
\begin{aligned}
S= & \int_{0}^{\pi} \sin \theta d \theta P_{2} \exp \left(-\frac{a}{k T} S P_{2}\right) \\
& \times\left[C_{1}+C_{2} \int_{0}^{\theta} d x \exp \left(\frac{a S}{k T} P_{2}(x)\right)\right] .
\end{aligned}
$$

In the case $C_{1}=1 / \int_{0}^{\pi} \exp \left(-a S P_{2} / k T\right) \sin \theta d \theta$, and $C_{2}=0$, we can obtain from the (20)

$$
\begin{aligned}
S & \approx \frac{48}{751}\left[1+\sqrt{1+\frac{5257}{120}\left(\frac{T_{c}-T}{T_{c}}\right)}\right] \\
& \approx 0.128+1.4\left(\frac{T_{c}-T}{T_{c}}\right) .
\end{aligned}
$$

From Eq. (21), we see that the first-order phase transition takes place which occurs not exactly at the temperature $T_{c}$, but at a slightly different temperature $T^{*}$ (it can be calculated that $T^{*} \approx 1.09 \cdot T_{c}$ ). The situation for $A=0$ at $T<T_{c}$, when a certain weak anisotropy is present in plane $x z$, is shown in Fig. 3(d).

Function $\delta(\theta, t)=y(\theta) \exp (-\lambda t)$ is found from the equation

$$
\begin{aligned}
-\frac{\lambda}{D} y= & \frac{\partial^{2} y}{\partial \theta^{2}}+\frac{a}{k T} \frac{\partial}{\partial \theta}\left[\frac { \partial P _ { 2 } } { \partial \theta } \left(y \int_{0}^{\pi} P_{2} f_{0} \sin \theta d \theta\right.\right. \\
& \left.\left.+f_{0} \int_{0}^{\pi} P_{2} y \sin \theta d \theta\right)\right] .
\end{aligned}
$$

For relatively small $S$, we obtain the following results from Eq. (22)

$$
y(\theta) \approx C \cos 2 \theta
$$

$$
\frac{\lambda}{D} \approx 4\left(1-\frac{T_{c}}{T}\right)+\frac{1}{5}\left(\frac{a}{k T}\right)^{2} S+\frac{23}{400}\left(\frac{a}{k T}\right)^{3} S^{2}
$$

Thus, we see that $\lambda$ depends on temperature, $\lambda\left(T=T_{c}\right)$ $\approx 0.54 \cdot D$, and the value of $\lambda$ increases with temperature decreasing [Eq. (23) includes the higher powers of $S$ when the order parameter is large].

\section{The case: $T<T_{c}$ and $A \neq 0$, but $A$ and $S$ are small at $t \rightarrow \infty$}

Since, in this case, the favorable director orientation is perpendicular to the light polarization, it is convenient to use Eq. (14b), where $\theta_{0}=\pi / 2, E_{\text {eff }}=0$, and $1 \gg A>0$. Using Eqs. (3) and (18), we can find that

$$
\begin{gathered}
f_{0}=\frac{1}{\int_{0}^{\pi} \sin \theta d \theta \exp \left[\left(-\frac{a S}{k T}+\frac{1}{3} A\right) P_{2}\right]} \\
\times \exp \left[\left(-\frac{a S}{k T}+\frac{1}{3} A\right) P_{2}\right], \\
S=\frac{1}{2} \int_{0}^{\pi} \sin \theta d \theta P_{2} f_{0} .
\end{gathered}
$$

Using Eqs. (24), we obtain the relation

$$
\varepsilon S \approx 1.56\left(5 S+\frac{1}{3} A\right)^{3}-\frac{1}{35}\left(5 S+\frac{1}{3} A\right)^{2}-\frac{1}{15} A,
$$




$$
\varepsilon=\frac{T_{c}-T}{T_{c}} \ll 1 .
$$

For the small values of $\varepsilon$ and $A$, Eq. (25) has the solution

$$
S \approx 0.128+1.4 \varepsilon+0.7 \cdot A,
$$

and Eq. (22) results in the value

$$
\frac{\lambda}{D} \approx 0.54+0.3 \varepsilon+2.5 \cdot A .
$$

If $\varepsilon$ is larger than $A$, then we see from $(25)$ that $S \approx \sqrt{7 \varepsilon} / 5$, with a correction of the order of $A / \varepsilon$. Equation (26a) shows that the saturation value of $S$ considerably increases with the increase of the parameter $A$. Equation (26b) shows the faster increase of the order parameter $S$ with time for the higher values of $\varepsilon$ and $A$.

The described situation is shown in Fig. 3(c), where a weak effective field is included to produce the $z$ orientation of director by the light action. The corresponding numerical results for the time dependence of the relative order parameter $s(t)$ are shown in Fig. 4. If $T<T_{c}$, then the characteristic relaxation time of $S$ decreases and the saturation value of $S$ increases for smaller temperatures, and in this case the parameter $-|a| / k T$ becomes larger (Fig. 4). After switching the light off, the order parameter rapidly becomes equal to the saturation values defined by temperature.

\section{F. Effect of external field to the order parameter}

In the films consisting of azo-dye molecules, the initial macroscopic orientational ordering is absent, due to the procedure of preparation of the films, even at low temperatures (at $T<T_{c}$ ). In fact, such a film is frustrated on many small areas of different sizes; the areas can possess average orientations which are occasional in the plane of the film. This means that the distribution function of molecular orientation $f(\theta, t)=$ const $=1 / 2$ before the light action. During the light exposure, finite sizes of the ordered areas are favorable for more rapid macroscopic ordering of the film, but the boundaries between differently oriented areas can slow down the process of photoaligning. We do not include these complicated features of a real film into the model consideration to simplify the solution of the problem. In the case where such azo-dye areas with initial occasional director orientations exist, we should use the equation of diffusion (1) with potential (14b) and nonisotropic distribution $f_{0}(\theta) \neq$ const. The initial director orientation $\theta_{0}$ and effective field $E_{\text {eff }}$ can be fixed, e.g., by the area boundaries.

Let us suggest that an effective field tends to align the director at the angle $\theta=\theta_{0}=0$, while the light action is orienting the director at the angle $\theta=\pi / 2$. If $T \approx T_{c}$, then for sufficiently small values of $E_{\text {eff }}$ and $I$ from Eq. (1) and potential $U$ given by Eq. (14b), we can obtain the order parameter $S$ from the equation

$$
\begin{aligned}
\varepsilon S \approx & 1.56 \cdot\left(5 S+\frac{1}{3}(B-A)\right)^{3}-\frac{1}{35}\left(5 S+\frac{1}{3}(B-A)\right)^{2} \\
& -\frac{1}{15}(B-A)
\end{aligned}
$$

where $\varepsilon \equiv\left(T_{c}-T\right) / T_{c} \ll 1, B \equiv \chi E_{\text {eff }}^{2} / k T_{c} \ll 1$.
Though in expansions used the parameters $A$ and $B$ are assumed to be small, the obtained results are approximately correct when magnitude $|B-A| \sim 1$, and we have the estimate

$$
S \approx \frac{1}{5}(B-A)^{1 / 3}, \text { if } 1>|B-A|>0.1 .
$$

Equation (28) shows that, for sufficiently large values of $B$, the value of $S$ is positive, i.e., the molecular orientation is determined by the effective with respect to the absorbing molecule external field. However, for sufficiently large values of $A$ the order parameter $S$ is negative, i.e., the molecular orientation is defined by the light action. These situations are illustrated schematically by Fig. 3(e) and 3(f). For a weak effective field the molecular orientation is induced by the light exposure [Fig. 3(e)]; for a strong effective field, the light is noneffective, and the molecular orientation is dictated by the field $\mathbf{E}_{\text {eff }}$ [Fig. 3(f)].

If the difference $|B-A|$ is small, i.e., $(B-A) \rightarrow+0$, then we obtain from Eq. (27) that

$$
S \approx 0.128+0.7(B-A) .
$$

When the value $(B-A)$ changes its sign and $(B-A) \rightarrow-0$, we obtain, instead of Eq. (29), the new solution of Eq. (28)

$$
S \approx-\frac{1}{5} \sqrt{\frac{7}{3}(A-B)} .
$$

Equations (29) and (30) show that the light-aligning process cannot reorient the dye molecules at weak light intensity: the threshold for light intensity is determined by the relation $A$ $=A_{c}=B$. The value of order parameter changes by a jump, and the change in sign of the order parameter takes place at this threshold.

\section{G. Approximation of "frozen" potential}

When the value of the relative exposure energy $A$ (2) is not small and the initial isotropic angular distribution function $f_{0}=1 / 2$ is highly distorted, we have to use numerical methods to find the solutions of the diffusion equation (1). One of the interesting possibilities here is to consider the molecular interaction potential in the form

$$
\begin{gathered}
\Phi=\frac{1}{2} A \cos ^{2} \theta+\frac{a}{k T}\left\langle P_{2}\right\rangle P_{2}(\theta), \\
\Phi=\frac{a}{k T}\left\langle P_{2}\right\rangle^{f} P_{2}(\theta),
\end{gathered}
$$

where (31a) and (31b) corresponds to the exposure process and relaxation, respectively, and $\langle P\rangle^{f}$ is the "frozen" order parameter during the relaxation process $(A=0)$

$$
\langle P\rangle^{f}=\left\langle P_{2}\right\rangle^{\text {off }}=\left\langle P_{2}\right\rangle\left(t_{\text {off }}\right),
$$

and $t_{\exp }=t_{\text {off }}$-exposure time. This means that the coefficient $a / k T\left\langle P_{2}\right\rangle^{f}$ before the term $P_{2}$ in (31) remains constant during the relaxation of the order parameter. The saturated value of the order parameter $\left.\left\langle P_{2}\right\rangle\right|_{t \rightarrow \infty}$ increases with the exposure time, as the coefficient $a / k T\left\langle P_{2}\right\rangle^{f}$ becomes larger for the higher values of $\tau$ exp. The situation is really confirmed in our experiment (see below). 


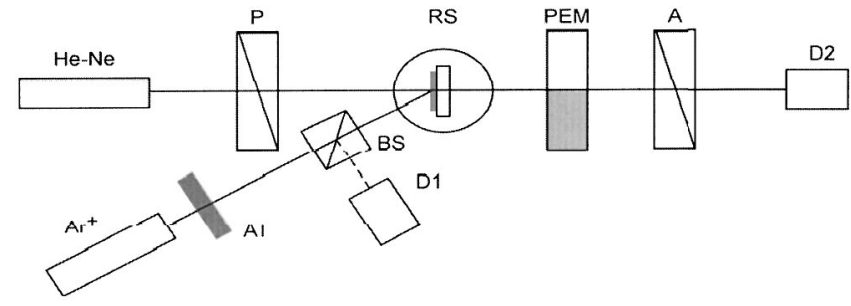

FIG. 5. Experimental setup. PEM is the photoelastic modulator; $\mathrm{P}, \mathrm{A}$ are the polarizer and analyzer, respectively; RS is the rotatory stage with SD film; AT is the attenuator; D1, D2 are the detectors; $\mathrm{Ar}^{+}, \mathrm{He}-\mathrm{Ne}$ are the lasers; BS is the beam splitter.

\section{EXPERIMENT}

The purpose of our experimental investigations was to measure uv light-induced birefringence, which is proportional to an order parameter of a photoaligning substance. We used in experiment the photosensitive sulfuric azo-dye SD (Fig. 1). The azo-dye was dissolved in $N$, $\mathrm{N}$-dimethylformamide at a concentration of $1 \mathrm{wt} \%$ and then spin-coated on ITO-coated glass substrate at $3000 \mathrm{rpm}$ for $30 \mathrm{~s}$. The coated substrate was cured for $15 \mathrm{~min}$ at $T$ $=45^{\circ} \mathrm{C}$. This procedure provides a thin, homogeneous solid dye film onto the ITO-glass surface. For accurate in situ measurement of small, thin film phase retardation $\delta$, a setup has been constructed (Fig. 5) [30,31]. It was based on a photoelastic modulator to measure the phase retardation value with an accuracy of $\pm 0.001 \mathrm{rad}$. SD birefringence was measured in-situ during the uv irradiation process (Fig. 5). The pump beam for inducing of optical anisotropy in SD film was provided by $\mathrm{Ar}^{+}$laser $(\lambda=457 \mathrm{~nm})$, which has a linear polarized light at the output with the power $W$ $=10 \mathrm{~mW} / \mathrm{cm}^{2}$. The output power was controlled by a special set of attenuator plates (AT). The probe beam, which comes from He-Ne laser $(\lambda=632.8 \mathrm{~nm})$, passed through crossed Glan-Thompson polarizers ( $\mathrm{P}$ and $\mathrm{A}$ ), the substrate with $\mathrm{SD}$ film, mounted on a rotatory stage (RS), and photoelastic modulator (PEM). The transmitted probe light intensity $I$ was modulated by PEM with a frequency $\Omega=50 \mathrm{kHz}$ and detected by semiconductive photodetector (D2).

The time dependence $I(t)$, recorded during several periods of light modulation, was transferred to computer for further treatment. In an ideal case, the PEM modulates the light according to the following law [30]:

$$
I_{\mathrm{id}}=\frac{1}{2}\left[1-\cos \left(\delta+A_{0} \cos (\Omega t)\right)\right],
$$

where $\Omega$ is a modulation frequency, $A_{0}$ is peak- and $\delta$ is sample phase retardation. In experiment, due to a residual birefringence of PEM, nonideal polarizers, and multiple reflections the background signal exists, and the registered signal is usually shifted in time. Due to these reasons, the following formula was used to describe the experimental data:

$$
I=I_{b}+I_{0} \frac{1}{2}\left\{1-\cos \left[\delta+A_{0} \cos \left(\Omega t+\alpha_{\varphi}\right)\right]\right\},
$$

where in addition to (33) $I_{b}$ is a background intensity, $I_{o}$ an incident intensity, and $\alpha_{\varphi}$ a phase shift. The typical experimental dependence of the transmitted light is shown in Fig. 6

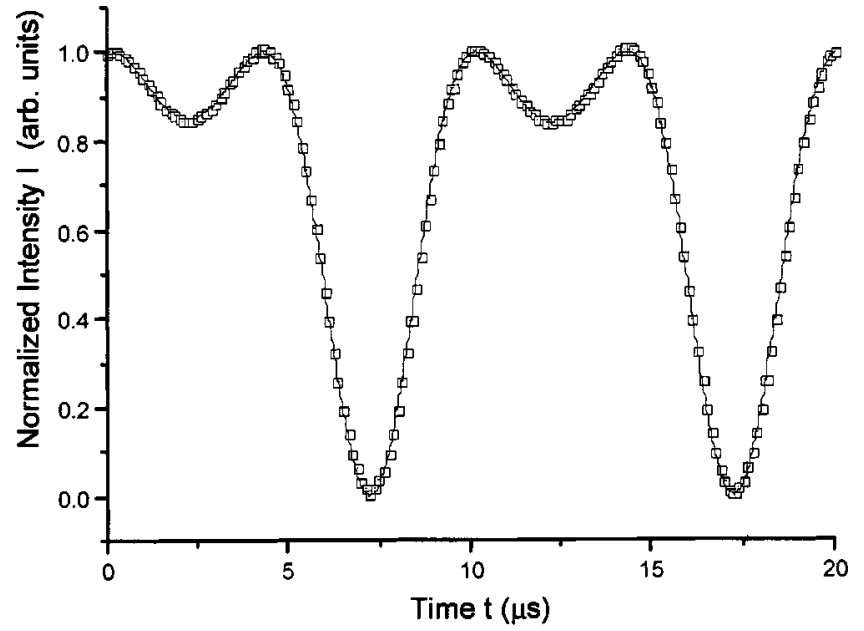

FIG. 6. Typical time dependence of the transmitted light intensity $I$. The experimental data are shown by squares, the fitting curve by solid line. The experiment was made using the setup shown in Fig. 5.

by squares. The peak phase retardation can be fixed by means of PEM controller. The sample retardation $\delta$ was determined by fitting the experimental data using (34) (solid line in Fig. 6), other parameters were approximately known from the experimental conditions and were corrected during the fitting procedure. The residual birefringence $\delta_{\text {res }}$ was measured before and after the experiment; the former was subtracted from the experimental data to obtain the absolute value of $\delta$. The change of $\delta_{\text {res }}$ during the time of the experiment was within the error limits. This procedure allows us to measure the phase retardation value more accurately than in the method based on measuring the first and second harmonics of photodetector signal (see Ref. 30 and references therein).

\section{DISCUSSION}

\section{A. The photoinduced birefringence (static case)}

To explain the experimental dependence of the photoinduced birefringence, we suggest the model of the rotational diffusion of the azo-dye molecules under the action of a polarized uv light, which we have described above (Sec. II C). The photoinduced phase retardation $\delta=2 \pi \Delta n d / \lambda$ ( $d$ $\approx 10 \mathrm{~nm}$ is the azo-dye layer thickness, $\lambda=632.8 \mathrm{~nm}$ ) is directly proportional to the relative order parameter $s$ of the azo-dye layer

$$
\delta=\frac{2 \pi \Delta n d}{\lambda}=\mathrm{k} s
$$

where $\mathrm{k}$ is a coefficient proportional to the anisotropy of the molecular polarizability of the azo-dye molecule [32].

The dependence of the phase retardation versus exposure time for different powers $W$ of pump $\mathrm{Ar}^{+}$laser is shown in Fig. 7. The size of the uv irradiated spot on the substrate was $2 \mathrm{~mm}$ in diameter. The thickness uniformity was controlled by measuring the phase retardation in different points of the sample. The difference in phase retardation does not exceed 


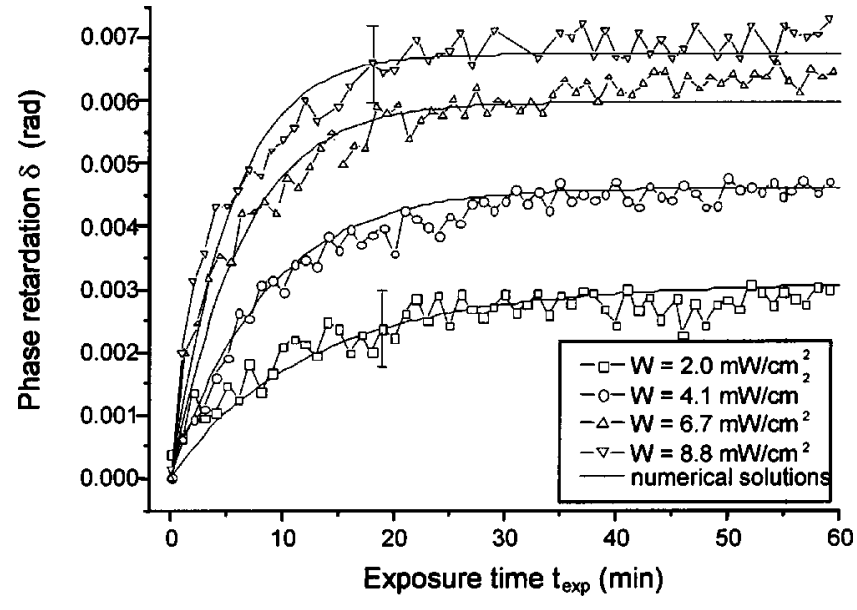

FIG. 7. Dependence of the photoinduced phase retardation $\delta$ on exposure time $t_{\exp }$ for various powers $W$ of the activated uv light. Solid lines indicate the numerical solution of the diffusion model (4) and (5).

the experimental error of $\pm 0.001 \mathrm{rad}$. The monotonous increase of the photoinduced phase retardation $\delta$ from zero level at $t=0$ (isotropic state) up to the saturation value $\delta_{0}$ (anisotropic state) was observed. The experimental values are in good agreement with the results of our numerical calculations, using the model (4) and (5), shown in Fig. 7 by solid lines. The saturation photoinduced phase retardation level was found to be proportional to the power of uv illumination $W$, which is in qualitative agreement with our analytical estimations (9) and (11) and the results of the numerical calculations (Fig. 8). The initial rate of the increase of the photoinduced birefringence should also establish a linear dependence (13). The derivatives $(d \delta / d t)_{t=0}$ were calculated from experimental data (Fig. 7). The numerical results, following from the model of orientational diffusion of the azodye molecules in the field of a uv-polarized light (4) and (5), are in good agreement with our experimental data (solid lines in Figs. 8 and 9) for

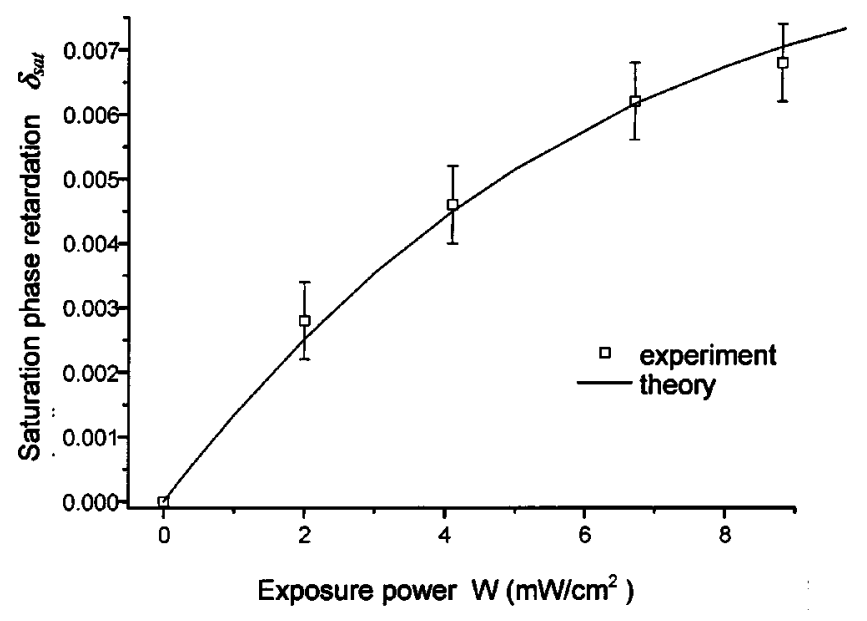

FIG. 8. Dependence of the relative saturation phase retardation $\delta_{\text {sat }}$ on power $W$ of the activated uv light. Solid lines indicate the numerical solution of the diffusion model (4) and (5).

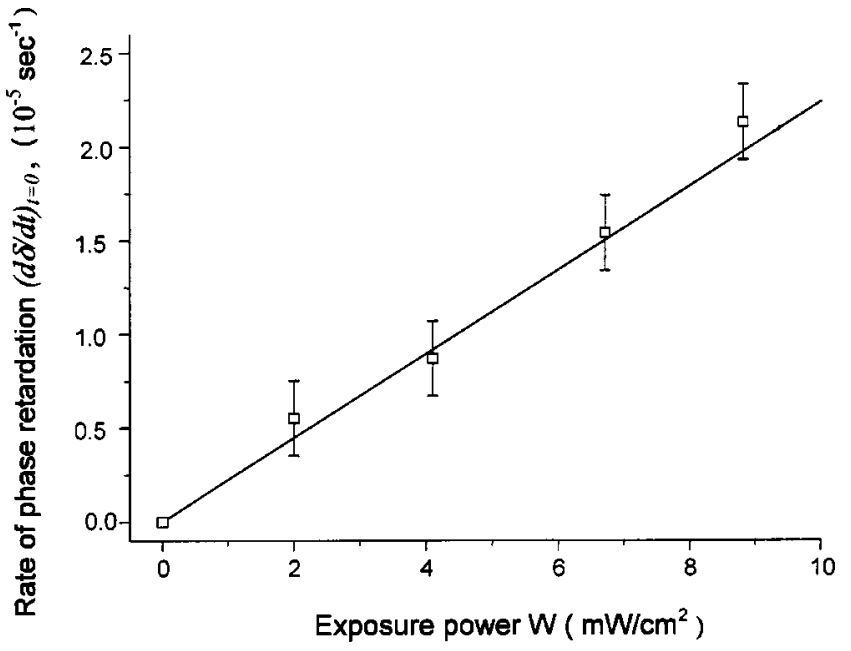

FIG. 9. Dependence of the initial rate of the photoinduced phase retardation increase $(d \delta / d t)_{t=0}$ on power $W$ of the activated uv light. Solid lines indicate the numerical solution of the diffusion model (4) and (5).

$$
\mathrm{k}=0.01, C=A / W=0.1 \mathrm{~m}^{2} / \mathrm{W}, \quad D=0.00026 \mathrm{~s}^{-1} .
$$

Using these data, we can determine the relative order parameter $s$ (9) and (11). For example, the relative order parameter according to the saturation level for irradiation power $W$ $=8.8 \mathrm{~mW} / \mathrm{cm}^{2}$ is $s=0.67$ and for $W=2.0 \mathrm{~mW} / \mathrm{cm}^{2}-s=0.3$ (Fig. 8). The saturation value of the phase retardation $\delta_{\text {sat }}$ shows a nonlinear increase with the power of the activated uv light beam (Fig. 8). The linear proportionality $s \approx W$ is observed only for sufficiently small values of W (Fig. 9). However, the exact numerical solution of the diffusion model (4) and (5) provides much better agreement (solid line in Fig. $8)$.

\section{B. Relaxation of the order parameter, taking into account the molecular interaction}

If we neglect the molecular interaction, the diffusion model (4) and (5) describes only the experimental data concerning the process of saturation of the order parameter with time (Figs. 7-9): the saturation value increases for the higher power (Fig. 8) as well as the rate of the saturation (Fig. 9). However, after switching the activated light off, the model predicts the exponential relaxation of the order parameter with time. Such a rapid decrease contradicts the experimental results, which show a new finite saturation level for a long time (Fig. 10). The experimental data in Fig. 10 show that, after switching the light off, the normalized phase retardation relaxes to a certain nonzero level dependent on temperature. The saturation level decreases with temperature, which is in good agreement with our estimations (21) and the results of the numerical calculations (Fig. 4). Taking into account the experimental data in Fig. 10, we evaluated the parameters $T_{C}$ and $a$ for the diffusion model (3) and (14a): $T_{C} \approx 453 \mathrm{~K}$, $|a|=5 k T_{C} \approx 3.1 \times 10^{-20} \mathrm{~J}$.

We have measured the relaxation dependencies of SD azo-dye samples for the same intensity of light, but different 


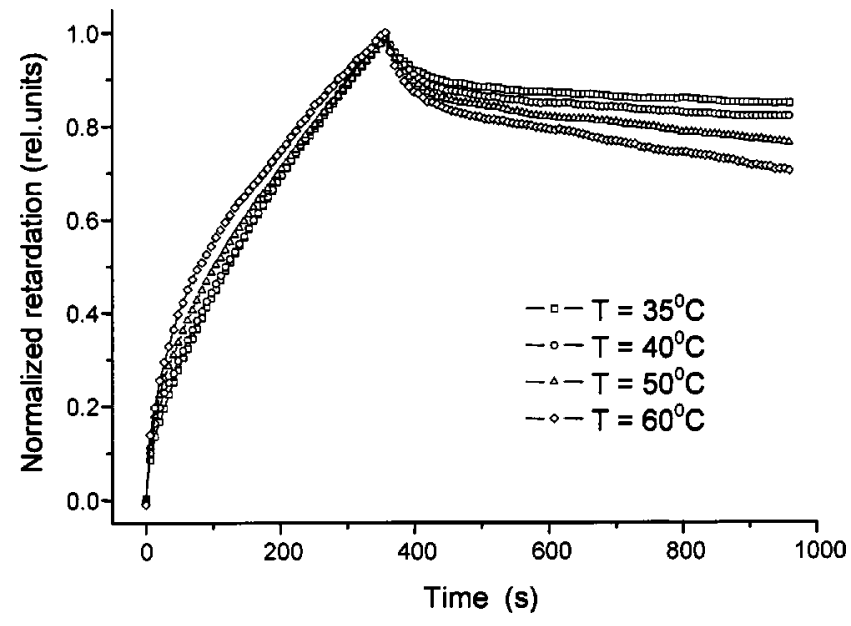

FIG. 10. Dependence of the normalized photoinduced phase retardation of azo-dye on time at various temperatures. The exposure energy was $40 \mathrm{~mJ} / \mathrm{cm}^{2}$ and the exposure time was $6 \mathrm{~min}$.

exposure times (Fig. 11). The sample was illuminated by linear polarized light during some time $\left(t_{\text {exp }}\right)$, and after this the uv source was switched off, but the registration of the phase retardation was continued up to the time when changing of the phase retardation $\delta$ was not observed. The results for different illumination times are shown in Fig. 11 [33]. The above-mentioned models (4) and (5) (molecular interaction is neglected) and the modified interaction potential (14b) cannot explain the order parameter relaxation, observed in our experiment (Fig. 11). Thus, we decided to use the approximation of the "frozen" potential (31a) and (31b) and make a fitting of the experimental curve by solving the problem (1) and (31) in numerical calculations (solid lines in Fig. 11). It can be seen that for a certain values of $A=0.1, a / k T$ $=-5.5$, the approximation of the frozen potential (31) provides a good coincidence between the theory and experiment (Fig. 11).

\section{CONCLUSION}

The model of the rotational diffusion of the azo-dye molecules under the action of a polarized uv light was used to explain the formation of the photoinduced order in azo-dye layers. A diffusion mechanism was proposed by us and does not involve any photochemical or structural transformation of azo-dye molecules. The model uses the diffusion equation with the specific potential, which characterizes the interaction of the molecule and the activated light. We consider both the approximations of negligible and strong molecular interaction during the process of the reorientation under the action of a polarized light. A certain critical temperature phase transition $T_{c}$ was predicted: for $T<T_{c}$ the order parameter of the azo-dye layer relaxes to nonzero value when the light

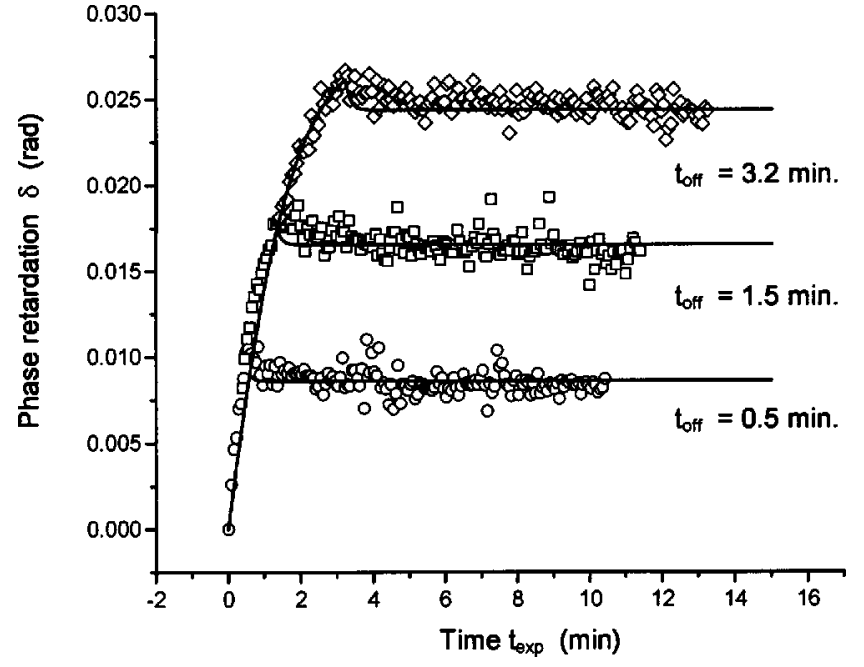

FIG. 11. Relaxation of SD azo-dye layer birefringence $\delta$ after switching off the activated light for different exposure times $t_{\text {off }}$ shown in Ref. [33]. The results of the numerical approximation (1) and (31) using the frozen potential (31) are shown by solid lines for $A=0.1$ and $a / k T=-5.5$.

field is switched off. We constructed an experimental setup, based on a photoelastic modulator, that allows to make accurate in situ measurements of the phase retardation $\delta$ of thin film as a function of the exposure time $t_{\text {exp }}$ and exposure power $W\left(W / \mathrm{cm}^{2}\right)$. Fitting the experimental curves $\delta\left(t_{\exp }\right)$ for different power values $W$, we can estimate the coefficient of rotational diffusion $D$, azo-dye order parameter $S\left(t_{\text {exp }}\right)$, and other parameters of the rotational diffusion model. A good agreement with experiment was observed even in the case of negligible molecular interaction: (i) the initial rate of the birefringence $d \delta /\left.d t\right|_{t=0}$ is proportional to the illumination power; (ii) the birefringence increases with the exposure time and comes to the saturation level $\delta_{\text {sat }} ;$ (3) the saturation value of birefringence $\delta_{\text {sat }}$ increases with the intensity of the activated light and is well described both by the analytical and numerical estimates.

To find nonzero values of the order parameter of the azodye layer after relaxation, we used the approximation of the frozen potential, which "remembers" the final value of the averaged order parameter at the moment when the light is switched off. A good agreement with the observed experimental value was found. The diffusion model of azo-dye reorientation in polymer matrix is in progress and will be published elsewhere.

\section{ACKNOWLEDGMENT}

This research was partially supported by RGC Grants HKUST6004/01E and HKUST6102/03E. 
[1] V. G. Chigrinov, V. M. Kozenkov, and H. S. Kwok, in Optical Applications of Liquid Crystals, edited by L. Vicari (Institute of Physics Publishing, Bristol and Philadelphia, 2003), p. 201.

[2] V. Chigrinov, E. Prudnikova, V. Kozenkov, H. S. Kwok, H. Akiyama, T. Kawara, H. Takada, and H. Takatsu, J. Soc. Inf. Disp. 11/3, 579 (2003).

[3] V. Chigrinov,E. Prudnikova, V. Kozenkov, H. Kwok, H. Akiyama, T. Kawara, H. Takada, and H. Takatsu, Liq. Cryst. 29, 1321 (2002).

[4] H. Benoit, Ann. Phys. (Paris) 6, 561 (1951).

[5] O. V. Stolbova, Dokl. Akad. Nauk SSSR 49, 84 (1963) (In Russian).

[6] M. Schönhoff, M. Mertesdorf, and M Löshe, J. Phys. Chem. 100, 7558 (1996).

[7] G. P. Bryan-Brown, and I. C. Sage, Liq. Cryst. 20, 825 (1996).

[8] J. Chen, D. K. Johnson, P. J. Bos, X. Wang, and J. L. West, Phys. Rev. E 54, 1599 (1996).

[9] H. G. Galabova, D. W. Allender, and J. Chen, Phys. Rev. E 55, 1627 (1997).

[10] J.-H. Kim, S. Kumar, and S.-D. Lee, Phys. Rev. E 57, 5644 (1998).

[11] A. Th. Ionescu, R. Barberi, M. Giocondo, M. Iovane, and A. L. Alexe-Ionescu, Phys. Rev. E 58, 1967 (1998).

[12] H. G. Galabova, D. W. Allender, and J. Chen, Phys. Rev. E 58, 3295 (1998).

[13] M. Hasegawa, Jpn. J. Appl. Phys., Part 2 38, L457 (1999).

[14] D. Andrienko, Y. Kurioz, M. Nishikawa, Y. Reznikov, and J. L. West, Jpn. J. Appl. Phys., Part 2 39, 1217 (2000).

[15] M. Dumont and A. El Osman, Chem. Phys. 245, 437 (1999).

[16] T. G. Pedersen, P. M. Johansen, N. C. R. Holme, P. S. Ramanujam, and S. Hvilsted, J. Opt. Soc. Am. B 15, 1120 (1998).

[17] T. G. Pedersen and P. M. Johansen, Phys. Rev. Lett. 79, 2470
(1997).

[18] S. P. Palto, N. M. Shtykov, V. A. Khavrichev, and S. G. Yudin, Mol. Mater. 1, 3 (1992).

[19] Linli Su, Bin Wang, J. West, and Y. Reznikov, Mol. Cryst. Liq. Cryst. Sci. Technol., Sect. A 359, 467 (2001).

[20] H. Takada, H. Akiyama, H. Takatsu, V. Chigrinov, E. Prudnikova, V. Kozenkov, and H. S. Kwok, SID'03 Digest, p. 620 (2003).

[21] Vladimir Chigrinov, Anatoli Muravski, Hoi Sing Kwok, Hirokazu Takada, Hidenari Akiyama, and Haruyoshi Takatsu, Phys. Rev. E 68, 061702 (2003).

[22] A. Morita and H. Watanabe, J. Chem. Phys. 70, 4708 (1979).

[23] Y.-H. Lee, D. Kim, S.-H. Lee, and W. G. Jung, J. Chem. Phys. 91, 5628 (1989).

[24] J. G. Kirkwood, and P. L. Auer, J. Chem. Phys. 19, 281 (1959).

[25] P. Debye, Phys. Z. 35, 101 (1934).

[26] D. J. Evans and Gary P. Morriss, Statistical Mechanics of Nonequilibrium Liquids (Academic, London-Tokyo, 1990).

[27] M. Doi, Faraday Symp. Chem. Soc. 18, 49 (1983).

[28] P. G. De Gennes, The Physics of Liquid Crystals (Clarendon, Oxford, 1974).

[29] L. Mederos and D. E. Sullivan, Phys. Rev. A 39, 854 (1989).

[30] B. Wang and T. C. Oakberg, Rev. Sci. Instrum. 70, 3847 (1999).

[31] H. S. Kwok, M. Khazimullin, and V. G. Chigrinov, Proc. SPIE 4799, 160 (2002).

[32] V. G. Chigrinov, Liquid Crystal Devices: Physics and Applications (Artech-House, Boston-London, 1999).

[33] M. Khazimullin, V. Chigrinov, and H. S. Kwok, ASID'02 Digest, p. 339 (2002). 